\title{
Understanding the Role of Molecular Diffusion and Catalytic Selectivity in Liquid-Phase Beckmann Rearrangement
}

\author{
Matthew E. Potter, ${ }^{\dagger}$ Alexander J. O’Malley, ${ }^{\ddagger},, \nabla$ Stephanie Chapman, ${ }^{\dagger}$ Julija Kezina, ${ }^{\dagger}, \|$
}

Stephanie H. Newland, ${ }^{\dagger}$ Ian P. Silverwood, ${ }^{\S, \perp}$ Sanghamitra Mukhopadhyay, ${ }^{\perp}$ Marina Carravetta, ${ }^{\dagger}$

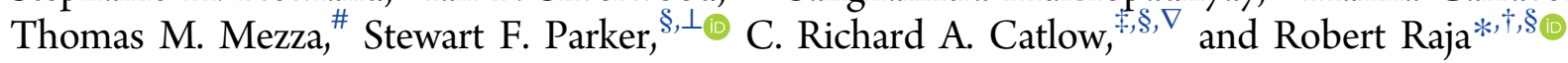

${ }^{\dagger}$ School of Chemistry, University of Southampton, University Road, Southampton SO17 1BJ, U.K.

${ }^{\ddagger}$ Department of Chemistry, University College London, 20 Gordon Street, London WC1H 0AJ, U.K.

${ }^{\S}$ UK Catalysis Hub, Research Complex at Harwell, Science and Technology Facilities Council, Rutherford Appleton Laboratory, Harwell Science and Innovation Campus, Oxon OX11 0QX, U.K.

"Analytical Sciences and Development, GSK, Medicines Research Centre, Gunnels Wood Road, Stevenage, Hertfordshire, SG1 2NY, U.K.

${ }^{\perp}$ ISIS Pulsed Neutron and Muon Facility, Science and Technology Facilities Council, Rutherford Appleton Laboratory, Harwell Science and Innovation Campus, Oxon OX11 0QX, U.K.

${ }^{\#}$ UOP LLC, A Honeywell Company, 25 East Algonquin Road, Des Plaines, Illinois 60017, United States

${ }^{\nabla}$ Cardiff Catalysis Institute, School of Chemistry, Cardiff University, Main Building, Park Place, Cardiff CF10 3AT, U.K.

\section{Supporting Information}

ABSTRACT: Understanding the role of diffusion in catalysis is essential in the design of highly active, selective, and stable industrial heterogeneous catalysts. By using a combination of advanced in situ spectroscopic characterization tools, particularly quasi-elastic and inelastic neutron scattering, we outline the crucial differences in diffusion modes and molecular interactions of active sites within solid-acid catalysts. This, coupled with 2D solid-state NMR and probe-based FTIR spectroscopy, reveals the nature of the active site in our SAPO37 catalyst and affords detailed information on the evolution of solid-acid catalysts that can operate at temperatures as low as $130{ }^{\circ} \mathrm{C}$, for the Beckmann rearrangement of cyclohexanone oxime to $\varepsilon$-caprolactam (precursor for Nylon-6). The

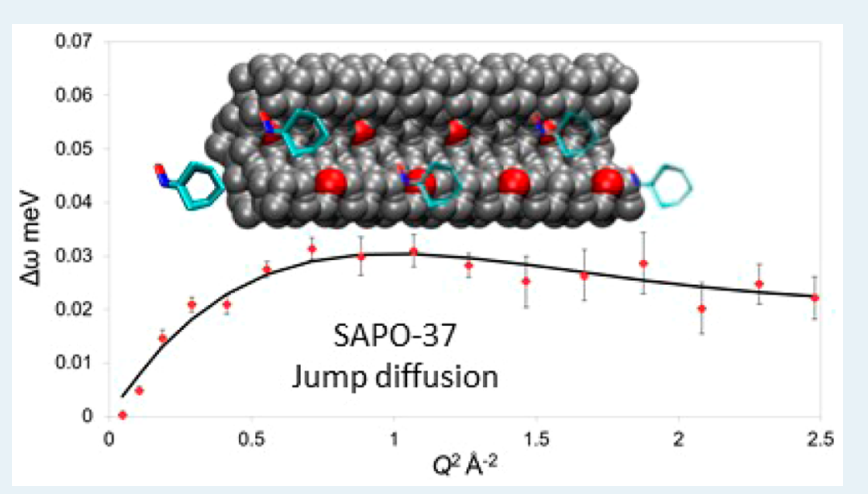
versatility of this approach leads to structure-property correlations that contrast the dynamics of the diffusion process in the different materials studied. Our results illustrate the power of these techniques in unravelling the interplay between active site and molecular diffusion in single-site heterogeneous catalysts, which can play a vital role in designing low-temperature, sustainable catalytic processes.

KEYWORDS: neutron scattering, catalytic diffusion, Beckmann rearrangement, heterogeneous catalysts, nylon-6, operando spectroscopy

\section{INTRODUCTION}

The ability to mimic the catalytic function of natural enzymes and tailor a robust structural environment, akin to the protein tertiary structure, to overcome complex mass transport and diffusion limitations, has long been a goal of synthetic chemists. ${ }^{1,2}$ Microporous zeotype architectures have proved particularly effective in facilitating diffusion in catalysts, where molecular transport can be controlled through a range of interconnecting channels and cages. ${ }^{3,4}$ It is further possible to devise synthetic procedures to target the formation of active sites that deliver a specific catalytic function (analogous to enzymes). ${ }^{5-10}$ This has enabled Brønsted and Lewis ${ }^{11-13}$ sites to be engineered within these frameworks, making them efficient and versatile solid acid catalysts within the petrochemical industry. ${ }^{5,14,15}$ Despite the range of zeotype frameworks known, only a few have been industrially exploited (MFI (ZSM-5), FAU (Zeolite-Y), CHA (SAPO-34), etc.). Unlike enzymes that operate at ambient to low temperatures, these inorganic analogues require higher temperatures, often in excess of $350{ }^{\circ} \mathrm{C}$, to overcome mass transport limitations and yield higher activity (potentially compromising selectivity, atom efficiency, and catalyst stability). ${ }^{16,17}$ The scope therefore for designing low-temperature, solid-acid catalysts for the Nylon-6 industry (predicted to be valued at 14 billion USD in 2019)

Received: December 22, 2016

Revised: $\quad$ March 3, 2017

Published: March 13, 2017 
Scheme 1. Typical Mechanistic Pathway for the Acid-Catalyzed Beckmann Rearrangement of Cyclohexanone Oxime

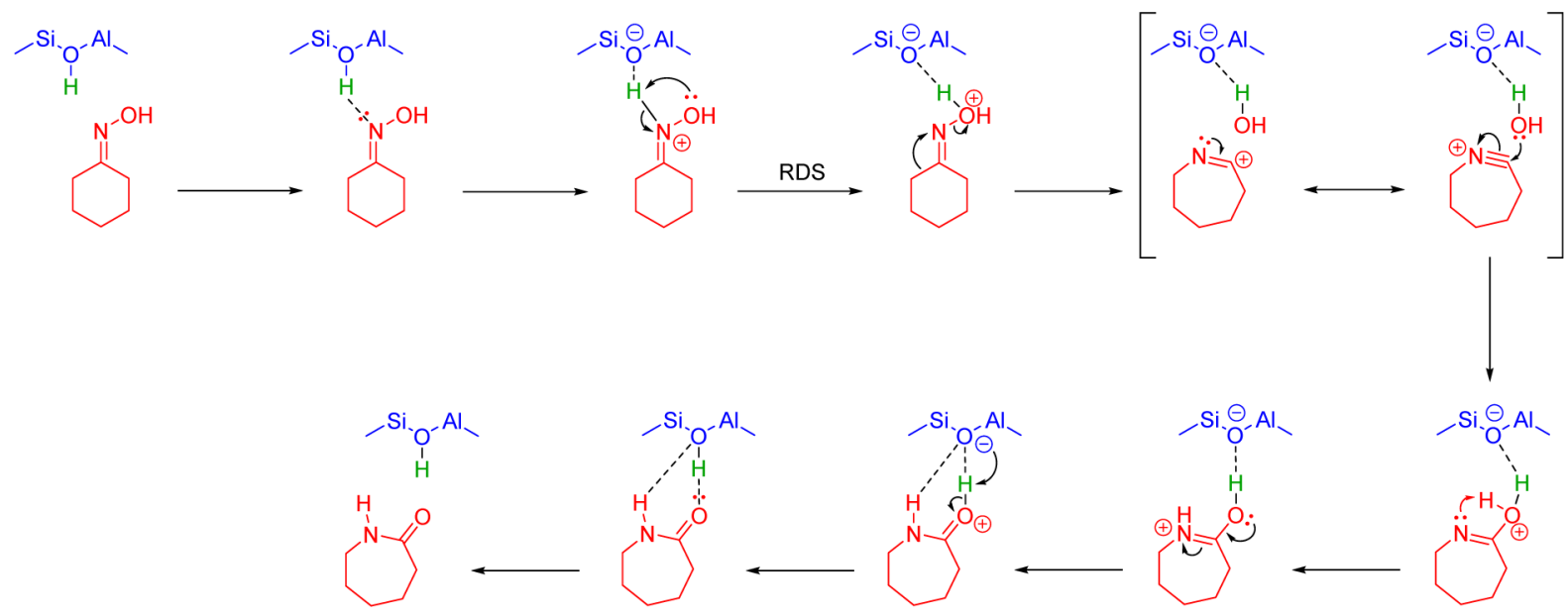

that can catalyze the Beckmann rearrangement of cyclohexanone oxime to $\varepsilon$-caprolactam (global production of 4.9 million tons in 2014) with high activities and selectivities is highly desirable. ${ }^{18}$

Despite the importance of diffusion processes in controlling reaction pathways and mechanisms, the role of diffusion and transport in nanoporous solids has typically been studied to a lesser extent than that of the active site. ${ }^{19}$ The dynamic behavior of cyclohexanone oxime within the channels of zeotype architectures in the Beckmann rearrangement is not clearly understood. Studies on ZSM-5 suggest the restrictive framework forces the reaction to occur on the catalyst's surface and in the periphery of the pore mouth, ${ }^{20-25}$ while the constricting micropores promote the formation of ring-opening byproducts. $^{22-25}$ Conversely, others have employed in situ infrared techniques to emphasize that the internal acid sites predominantly yield the lactam. ${ }^{24,25}$ Therefore, unsurprisingly, it is challenging to understand a reaction process while separating the influences of molecular diffusion and the nature of the active site. To facilitate a more holistic understanding, we present a combined catalytic and multitechnique characterization study (employing in situ techniques), investigating the catalyzed, liquid-phase Beckmann rearrangement of cyclohexanone oxime using a tailored SAPO-37 (aluminophosphate analogue of the zeotype FAU) architecture, with controlled acid site concentration. ${ }^{15}$

To ensure meaningful comparisons, parallel studies were also performed on Zeolite-Y (aluminosilicate analogue of the zeotype FAU), to facilitate detailed structure-property correlations and explore the interplay between active-site structure and molecular diffusion within the same FAU architecture. For the sake of completeness, we also probed the efficacy of the (conventionally used) ZSM-5 catalyst ${ }^{20,26}$ in the liquid-phase Beckmann rearrangement of cyclohexanone oxime to contrast the influence of pore aperture with our SAPO-37 analogue. In particular, we present the use of quasielastic neutron scattering (QENS), which has been shown to effectively characterize and quantify diffusion of confined hydrocarbons $\mathrm{s}^{27,28}$ and was recently used in tandem with vibrational spectroscopy to gain insight into key steps of the zeolite-catalyzed methanol to hydrocarbons process in commercial catalysts. ${ }^{28}$ Here we directly study the dynamics of the reactant, cyclohexanone oxime, to determine the nature of the diffusion in the liquid-phase Beckmann rearrangement which, coupled with inelastic neutron scattering (INS), solidstate 2D NMR, and probe-based FTIR spectroscopy, offers valuable insights into the specific role played by solid-acid sites in the mechanistic and reaction pathways (Scheme 1). ${ }^{21}$ The QENS study further supports our design rationale of combining a specific active site with a specific framework topology to create an effective catalyst, which affords near-quantitative yields of $\varepsilon$-caprolactam at temperatures as low as $130{ }^{\circ} \mathrm{C}$.

\section{EXPERIMENTAL METHODS}

QENS Methodology. All QENS measurements were performed using the high-resolution time-of-flight (TOF) instrument OSIRIS at the ISIS Pulsed Neutron and Muon Source, Chilton, Oxfordshire, U.K.. The analyzer crystals used were cooled pyrolytic graphite (PG002) with the energy transfers measured in a window of $\pm 0.61 \mathrm{meV}$. The energy resolution of this instrument with this configuration in nearbackscattering geometry is $24.5 \mu \mathrm{eV}$. The samples ( $4.5 \mathrm{~g}$ each in total) were transferred inside a glovebox, after drying, to cylindrical aluminum containers of annular geometry. The cells were placed in a CCR cryostat and a resolution measurement was taken at $30 \mathrm{~K}$. The measurements were then taken at 373 $\mathrm{K}$. The signal taken from an empty zeolite was taken preloading and then subtracted from the signal of the loaded zeolite so that only the signal from the oxime was measured.

Inelastic Neutron Scattering Spectroscopy. The SAPO37 was dried under a flow of $\mathrm{He}$ gas at $550{ }^{\circ} \mathrm{C}$ for $2 \mathrm{~h}$. A $1 / 10$ solid-state mixture of cyclohexanone oxime and SAPO-37 was prepared in a glovebox, under an argon atmosphere. The mixture was transferred to an aluminum-foil sachet, which was sealed in an aluminum can. The experiments were carried out using the high-resolution INS spectrometer MAPS at the ISIS Pulsed Neutron and Muon Source (Oxfordshire, U.K.). The cans were placed in a top-loading, closed-cycle refrigerator cryostat; all spectra were collected at $<30 \mathrm{~K}$ in order to minimize the Debye-Waller factor. Measurements were taken at neutron incident energies of $650 \mathrm{meV}$ ("A" chopper speed $600 \mathrm{~Hz}$ ), $250 \mathrm{meV}$ (“A” chopper speed $400 \mathrm{~Hz}$ ), and $100 \mathrm{meV}$ (“A” chopper speed $200 \mathrm{~Hz}$ ).

\section{RESULTS AND DISCUSSION}

The framework integrity of SAPO-37, Zeolite-Y, and ZSM-5 was determined via a range of techniques. Powder XRD patterns of the three samples (Figure S1 in the Supporting 

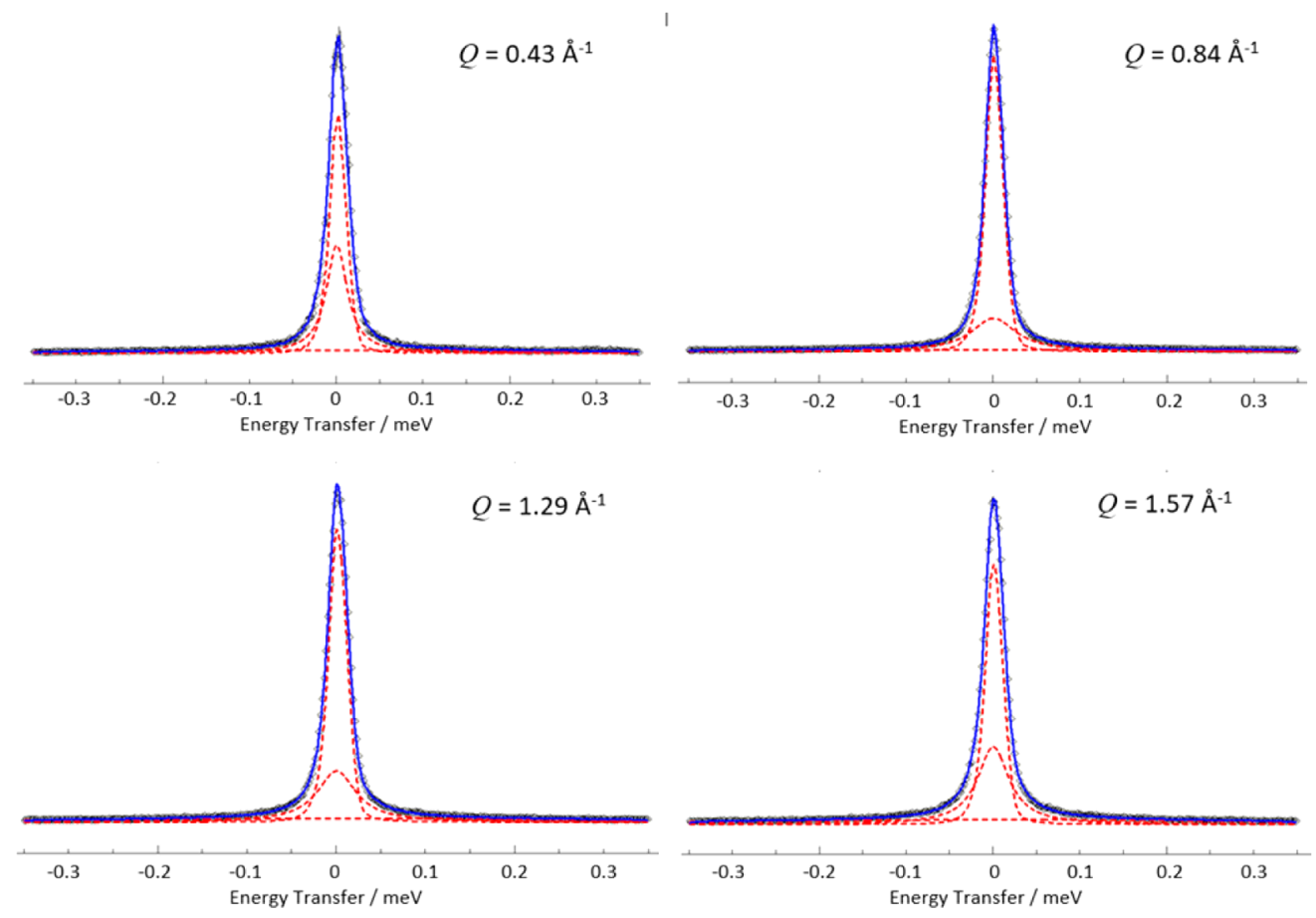

Figure 1. QENS spectra obtained for cyclohexanone oxime diffusing in SAPO-37 at four different $Q$ values at $373 \mathrm{~K}$, showing the total fit (blue), the constituent resolution, and Lorentzian and flat background functions (red).

Information) show that all materials are highly crystalline and phase-pure, suggesting an ordered structure with minimal defects. The crystallinities were compared with respect to a potassium iodide standard and showed that Zeolite- $Y$ and SAPO-37 have very similar levels of crystallinity (Figure S1). Particle size is also known to influence diffusion in porous materials, and scanning electron microscopy (SEM, Figure S2 in the Supporting Information) revealed the presence of uniform particles ( $1 \mu \mathrm{m}$ in size), in all three catalysts. The exterior of the particles appeared smooth and regular in all cases, suggesting similar levels of surface defects (Figure S2). Finally, $\mathrm{N}_{2}$ physisorption isotherms showed good agreement between the Zeolite-Y and SAPO-37 frameworks for surface area and micropore volume (Table S1 in the Supporting Information), which was in good agreement with the literature values. $^{29,30}$ It is important to note that similar levels of dopant species (Al for zeolites, Si for SAPO-37) were deliberately introduced in all three catalysts (Table S1), to ensure that meaningful comparisons can be established for contrasting the influence of the dopant substitution on active-site formation.

Quasi-elastic neutron scattering (QENS) was used to monitor the molecular motion of cyclohexanone oxime within the inorganic zeotypes, at temperatures $(373 \mathrm{~K})$ akin to those of the liquid-phase catalytic experiments (experimental limitations restrict the use of higher temperatures). A more rigorous discussion of QENS theory can be found in the Supporting Information and is summarized below. Hydrogen has the largest incoherent scattering cross-section of any element $^{31}$ and so is the primary contributor to the experimentally measured incoherent scattering function, $S(\mathbf{Q} \omega)$, allowing the oxime to be observed in the presence of the prominent inorganic matrix. Molecular motions perturb elastic neutron-matter interactions, causing quasi-elastic deviations that result in a quantifiable broadening of the elastic line. For isotropic diffusion, the broadening of $S(\mathbf{Q}, \omega)$ is described as a Lorentzian function, linking the half-width half- maximum (HWHM) of the quasi-elastic component $\Delta \omega(Q)$ to the self-diffusion coefficient $D_{s}$ and momentum transfer vector $Q\left(\AA^{-1}\right)$ between the incident and scattered neutron, where $\omega$ is neutron energy transfer $(\mathrm{meV}) .^{31-33}$

$$
\Delta \omega(Q)=D_{s} Q^{2}
$$

Different models are needed for non-Fickian behavior, such as the Chudley-Elliot model, ${ }^{33}$ where a molecule is temporarily immobilized before jumping to the next site. These models include derivable parameters such as residence time and jump distance. ${ }^{33}$ The QENS spectra of cyclohexanone oxime in ZSM-5 (Figure S3 in the Supporting Information) closely match the Gaussian instrument resolution function at lower $Q$ values, with no observable quasi-elastic component, indicating that no long-range translational diffusion is observable over the time and length scales accessible to the spectrometer at these temperatures. ${ }^{34}$ A minimal Lorentzian component is observed at the higher $Q$ values. ${ }^{35,36}$ As we cannot detect any broadening at low momentum transfers, it is difficult to say whether these broadenings may be associated with rotational motion or indeed conform to the higher $Q$ behavior of a jump diffusion model. Regardless, this component is too small for reliable analysis, and a spectrometer of higher resolution, able to detect motion over longer time scales, would be necessary to characterize or quantify any mobility in this system. However, the minimal quasi-elastic component indicates that any translational diffusion occurring would be much slower than can be detected here. ${ }^{37}$ It is plausible that the channel dimensions $(5.5 \AA)$ are too small to allow significant diffusion of the cyclohexanone oxime (sorbate) at these temperatures; thus, internal active sites cannot be accessed for this catalyst under these conditions. (It has been shown that such sites may be accessible under vapor-phase conditions $\left(>300{ }^{\circ} \mathrm{C}\right),{ }^{24,25}$ which is outside the scope of the current study.) 

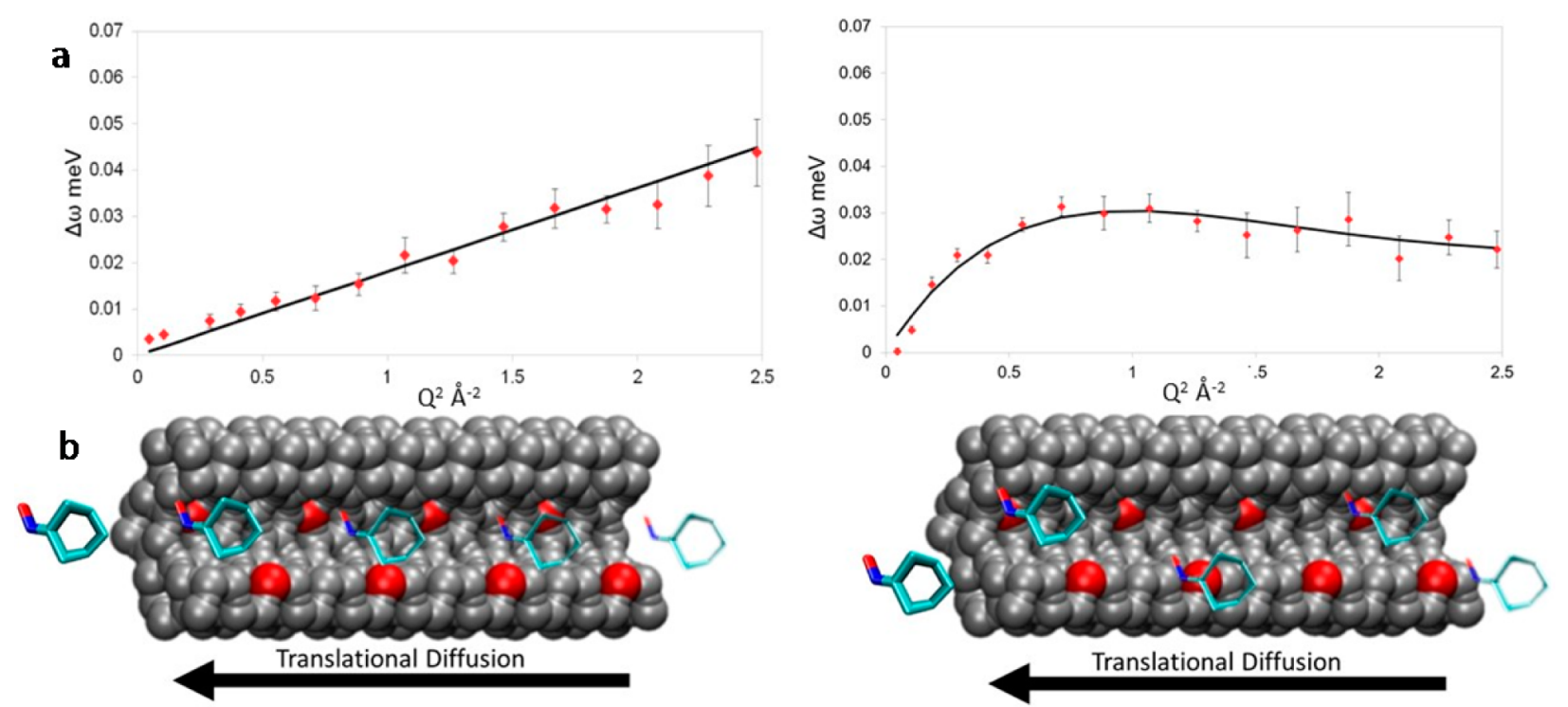

Figure 2. (a) Q dependence of the HWHM of the Lorentzian for Zeolite-Y (left) and SAPO-37 (right) at $373 \mathrm{~K}$. (b) Contrasting diffusion modes of cyclohexanone oxime flowing through a pore with active sites (red). The oxime moves straight through the pore when there is a smooth Fickian diffusion (left with Zeolite-Y) and the oxime shifts between active sites with a specific residence time during jump diffusion (right with SAPO-37).
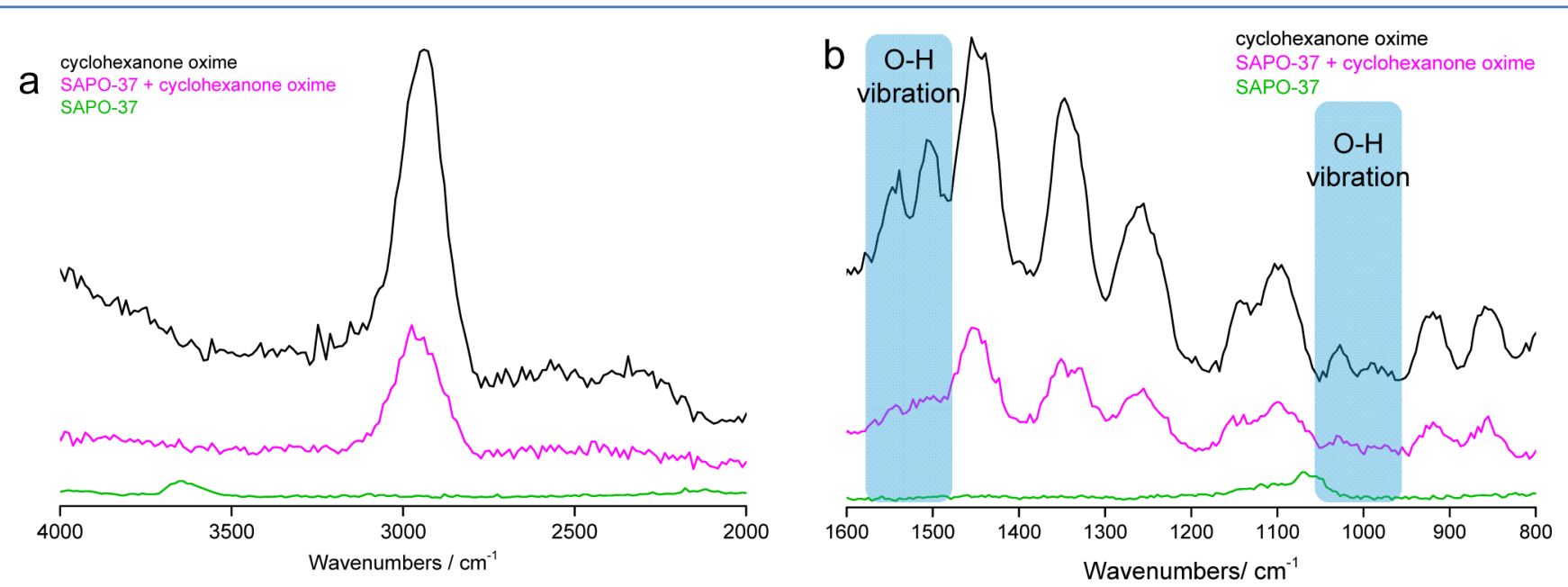

Figure 3. INS vibrational spectra showing the influence of oxime binding to SAPO-37: (a) $E_{\mathrm{i}}=650 \mathrm{meV}$, with the Brønsted acid peak in SAPO-37 $\left(3650 \mathrm{~cm}^{-1}\right)$ removed on addition of the oxime, showing the interactions between the two; (b) $E_{\mathrm{i}}=250 \mathrm{meV}$, showing the reduction in the $\mathrm{O}-\mathrm{H}$ vibration bands relative to the ring deformation modes.

Spectra obtained for the sorbate in both Zeolite-Y and SAPO-37 for four $Q$ values are depicted in Figure S4 in the Supporting Information and Figure 1, respectively. The error in the neutron data points in Figure 1 and Figures S3 and S4 in the Supporting Information are assigned on the basis of Poisson statistics. All spectra were fit with a resolution function, a single Lorentzian function describing the quasi-elastic component, and a flat background, suggesting that one dominant motion is present on the instrument time scale. The Lorentzian component obtained for Zeolite-Y (Figure S4) showed continuous broadening, whereas SAPO-37 only showed broadening up to a $Q$ value of $\sim 1.2 \AA^{-1}$ (Figure 1). The errors in the Lorentzian HWHM fits are assigned using a Monte Carlo method, where data sets are generated virtually within the neutron data point error bars and then fitted.

The HWHM broadening in Zeolite-Y shows a $D Q^{2}$ dependence, consistent with Fickian diffusion (Figure 2a), indicating that the oxime moves freely through the micro- porous framework (diffusion coefficient of $[2( \pm 0.18)] \times 10^{-10}$ $\left.\mathrm{m}^{2} \mathrm{~s}^{-1}\right) .^{38}$ The SAPO-37 $Q$ dependence is not linear (Figure 2b) but follows the Chudley-Elliot model for jump diffusion (a jump mechanism was previously observed for benzene in Na$\mathrm{Y})^{33}$ with a jump distance of $4.5 \AA$ and a residence time of $50 \mathrm{ps}$ (Figure 2). This leads to an extrapolated diffusion coefficient of $\left.[6.5( \pm 2.7)] \times 10^{-10} \mathrm{~m}^{2} \mathrm{~s}^{-1}\right)$. We note that while the higher $D_{\mathrm{s}}$ approximated from the jump model is an interesting observation, the different mechanisms from which these values are calculated and the significantly higher errors associated with the jump model mean that a direct comparison of the two values must be treated with caution. The observed jump distance $(4.5 \AA)$ matches the length of a window region within the faujasite structure, showing the sorbate residing in one supercage before jumping into a neighboring cage; this result suggests that the oxime is able to diffuse through the internal pores (at these low temperatures), accessing the internal acid sites. The tendency of the oxime to reside in certain areas of the 
SAPO-37 framework derives from the interactions with the internal acid sites. These interactions would potentially yield a greater production of lactam than in Zeolite-Y, which allows Fickian diffusion of the reactant (suggesting minimal interactions between the oxime and acid sites). The constrained nature of the motion may also be attributed to pore occlusion. Acid sites which are located at the pore mouths may interact with the oxime molecule, preventing it from accessing the pores. These findings were also independently verified by carrying out separate catalytic tests that mimic the experimental conditions and setup as outlined in Figure 2 (90\% catalyst, 10\% oxime, $373 \mathrm{~K}, 8 \mathrm{~h}$, solvent free, under a nitrogen environment) for all three catalysts. We did not observe any appreciable $(<5$ mol \%) conversion at $373 \mathrm{~K}$ in all three cases, which vindicates our conclusion that any QENS signal is predominantly due to the cyclohexanone oxime. The reactant has therefore a differing diffusion mechanism at this temperature via jump diffusion but does not react.

To understand better the change in diffusional behavior, an inelastic neutron spectroscopy study (INS) was performed. INS spectra are similarly dominated by modes involving hydrogen motion; therefore, the spectra largely reflect the hydrogenous component present. Figure $3 \mathrm{a}$ shows the $\mathrm{O}-\mathrm{H}$ stretching region of SAPO-37 (4000-2000 $\left.\mathrm{cm}^{-1}\right)$, before and after introduction of the oxime. Interestingly, the $\mathrm{O}-\mathrm{H}$ stretch of the framework hydroxyls $\left(3650 \mathrm{~cm}^{-1}\right)$ has been severely reduced. This demonstrates a significant interaction between the SAPO-37 and the oxime, in keeping with the observed jump diffusion in the QENS. Focusing on a lower energy range (1600-800 $\mathrm{cm}^{-1}$, Figure $3 \mathrm{~b}$ ) provides more detailed insights into the oxime-SAPO-37 interactions. On comparison of pure cyclohexanone oxime (black line, Figure $3 \mathrm{~b}$ ) with cyclohexanone oxime combined with SAPO-37 (pink line, Figure $3 \mathrm{~b})$, several peaks recede. Most notably this occurs in the $1600-1500 \mathrm{~cm}^{-1}$ region and the $1050-950 \mathrm{~cm}^{-1}$ region. These peaks are assigned as $\mathrm{O}-\mathrm{H}$ vibrations from DFT-based CASTEP calculations. ${ }^{39,40}$ The other peaks (attributed to C$\mathrm{H}$ vibrations through the same calculations) remain comparatively constant. The SAPO-37 O-H bend at $1060 \mathrm{~cm}^{-1}$ has receded, most likely upshifted due to hydrogen-bonding interactions with the oxime, and may be enveloped by the intense ring deformation modes surrounding it. This observation suggests that the oxime is indeed interacting with the acid sites of the SAPO-37 via the $\mathrm{OH}$ group of the oxime (as seen by the disappearance of both signals in the INS; Figure 3 ), in keeping with the suggested mechanism (Scheme 1).

The combined INS and QENS data reveal fascinating contrasts between the three catalysts. In ZSM-5, the reactant is unable to access the internal pores under these time scales, while in Zeolite-Y, the molecules diffuse freely through the pore and therefore do not interact with the solid-acid sites at $373 \mathrm{~K}$. In SAPO-37, the enhanced interaction, as seen through INS, (facilitated by the nature, type, and strength of acid centers within the internal pores, as discussed later) with these sites results in jump diffusion at lower temperatures, which is crucial for the enhanced selectivity of this catalyst, in the liquid-phase Beckmann rearrangement.

Solid-state MAS NMR spectroscopy probes the local structural environment of framework atoms. The bulk AlPO matrix of SAPO-37 was affirmed through ${ }^{31} \mathrm{P}$ (Figure S5 in the Supporting Information) and ${ }^{27} \mathrm{Al}$ MAS NMR (Figure 4 and Figure S6 and Table S2 in the Supporting Information). ${ }^{31} \mathrm{P}$ MAS NMR showed one well-resolved peak at $-27 \mathrm{ppm}$,
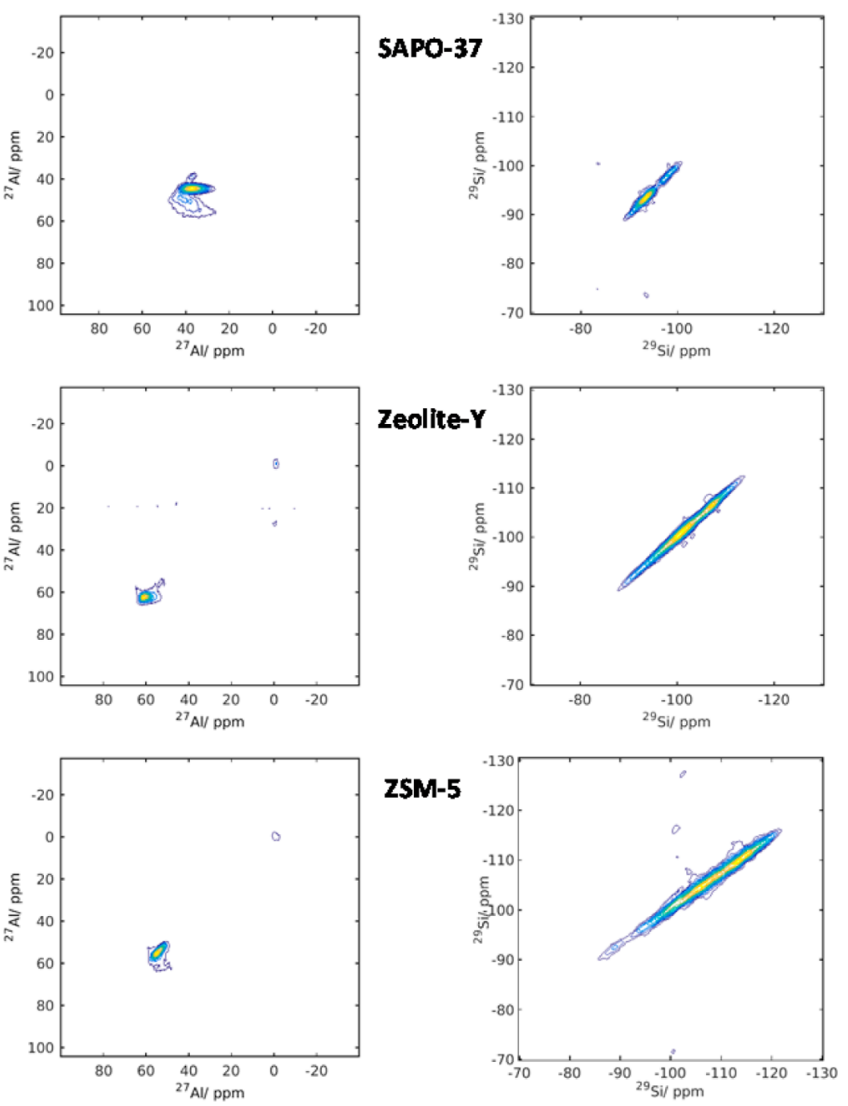

Figure 4. 2D MAS ${ }^{27} \mathrm{Al}$ and ${ }^{29} \mathrm{Si}$ NMR spectra for the zeotypes.

attributed to $\mathrm{P}(\mathrm{OAl})_{4}$. The $1 \mathrm{D}{ }^{27} \mathrm{Al}$ MAS NMR of SAPO-37 shows a broad peak at $33 \mathrm{ppm}$, with limited evidence of octahedral aluminum species at $\sim 0 \mathrm{ppm}$. The ${ }^{29} \mathrm{Si}$ nuclei show a broad signal, with an apex at $-91 \mathrm{ppm}$ (Figure S7 in the Supporting Information), suggesting that the majority of silicon has formed $\mathrm{Si}(\mathrm{OAl})_{4}$ species, confirming the incorporation of silicon into the material, ${ }^{41}$ via type II substitution (Si replacing P). ${ }^{7}$ D homonuclear MAS NMR (Figure 4) provides further resolution on the ${ }^{27} \mathrm{Al}$ and ${ }^{29} \mathrm{Si}$ NMR spectra. ${ }^{42}$ The broad $1 \mathrm{D}$ ${ }^{27} \mathrm{Al}$ peak is a combination of two signals, attributed to $\mathrm{Al}(\mathrm{OP})_{4}$ and $\mathrm{Al}(\mathrm{OP})_{3}(\mathrm{OSi})$, which arises from the isomorphous substitution of silicon into the framework. ${ }^{43}$ The additional sharp peak in the 3QMAS spectrum is small and is compatible with a tetrahedrally coordinated $\mathrm{Al}$, possibly a surface defect site, such as $\mathrm{Al}(\mathrm{OP})_{3}(\mathrm{OH})$ (Figure 4 and Table $\mathrm{S} 2$ in the Supporting Information). Similarly the ${ }^{29} \mathrm{Si}$ spectra were deconvoluted into two peaks at -91 and $-98 \mathrm{ppm}$, attributable to $\mathrm{Si}(\mathrm{OAl})_{4}$ and $\mathrm{Si}(\mathrm{OAl})_{3}(\mathrm{OSi})$, respectively, providing evidence for the formation of small silicon islands (Table S2). The ${ }^{29} \mathrm{Si}$ spectra of Zeolite-Y and ZSM-5 show two different environments, confirmed by $2 \mathrm{D}$ MAS NMR (Figure 4). The lower signal ( $-111 \mathrm{ppm}$ in ZSM-5 and $-106 \mathrm{ppm}$ in Zeolite-Y) can be attributed to different framework topologies of bulk $\mathrm{Si}(\mathrm{OSi})_{4}{ }^{44,45}$ The second signal (-105 ppm in ZSM-5 and $-101 \mathrm{ppm}$ in Zeolite-Y) is due to the presence of $\mathrm{Si}(\mathrm{OSi})_{3}(\mathrm{OH})$ and $\mathrm{Si}(\mathrm{OAl})(\mathrm{OSi})_{3}$ species. The latter confirms that aluminum has undergone isomorphous substitution and resides within the framework. ${ }^{44} \mathrm{Al}$ incorporation is further demonstrated through ${ }^{27} \mathrm{Al}$ NMR spectra for ZSM-5 and Zeolite-Y. Both species show a large fraction of frameworkincorporated $\mathrm{Al}$ atoms, as evidenced by signals in the region of 
54-62 ppm, which are attributed to $\mathrm{Al}(\mathrm{OSi})_{4}$ species, with a further signal at $0 \mathrm{ppm}$, attributed to extraframework octahedral Al sites (Figure 4), both in good agreement with published literature. ${ }^{46,47}$ The acid sites were investigated using $\mathrm{NH}_{3}$-TPD (Figure S8 and Table S3 in the Supporting Information) and low-temperature, CO probe FT-IR (Figure 5 and Figures S9-

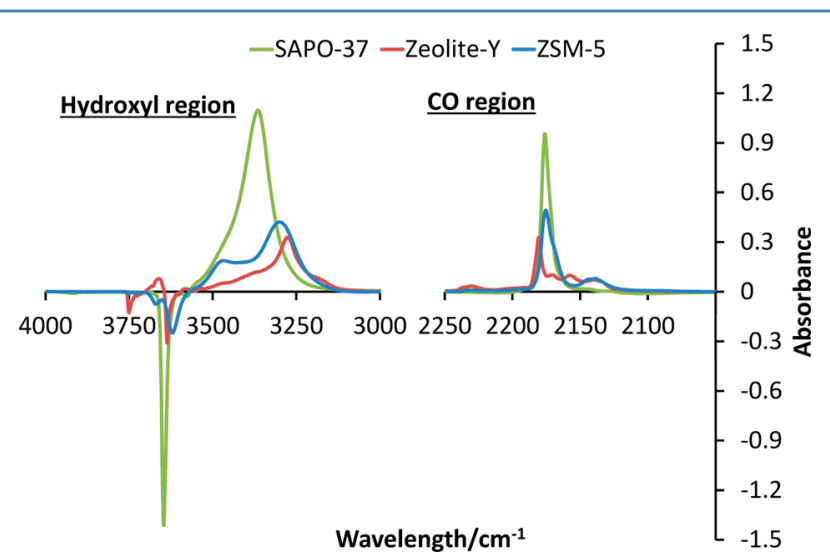

Figure 5. FT-IR difference spectra for low-temperature $\mathrm{CO}$ adsorbed on SAPO-37 (green), Zeolite-Y (red) ,and ZSM-5 (blue), showing the decline of hydroxyl groups and a shift to lower wavenumbers in the hydroxyl region, with an increase in the $\mathrm{CO}$ stretching region showing the binding to the Brønsted acid species.

S13 and Table S4 in the Supporting Information). Both zeolites show significant quantities of defect silanol species $\left(3750 \mathrm{~cm}^{-1}\right.$, Figure S9), which induce weak acidity, ${ }^{48,49}$ as confirmed from the $\mathrm{NH}_{3}$-TPD spectra of Zeolite-Y and ZSM-5 (silanol signal at $270{ }^{\circ} \mathrm{C}$, Figure S8). SAPO-37 does not have a $\mathrm{Si}-\mathrm{OH}$ feature, though minor amounts of $\mathrm{Al}-\mathrm{OH} / \mathrm{P}-\mathrm{OH}$ defect species are present (Figure S9). ${ }^{50}$ In agreement with the NMR, $\mathrm{Al}-\mathrm{OH}$ species are present to a small extent in Zeolite-Y and ZSM-5, though these are unlikely to influence the reactivity of the catalysts. FT-IR further confirms the presence of $\mathrm{Si}-\mathrm{OH}-\mathrm{Al}$ Brønsted acid species, from incorporation of the dopant atoms into the framework. The signal from Zeolite-Y and SAPO-37 is split into two characteristic bands, assigned to protons residing in supercages and sodalite cages of the faujasite system. Protons in ZSM-5 only occupy the one-dimensional channel; thus, a single $\mathrm{Si}-\mathrm{OH}-\mathrm{Al}$ band is observed. These findings indicate that the SAPO-37 catalyst is distinct from its zeolitic counterparts in possessing a higher fraction of isolated Brønsted acid sites, owing to the lack of silicon island formation, and is conspicuous for its lack of defect silanols, resulting in uniform acidity.

The proton band (hydroxyls) shifted on adsorption of $\mathrm{CO}$ (Figures S10 and S11 in the Supporting Information), where the magnitude of the shift correlates with acid site strength. Both zeolites possess stronger acid sites in comparison to SAPO-37, which was independently verified with $\mathrm{NH}_{3}$-TPD (Figure S8 in the Supporting Information). ${ }^{51}$ The CO stretching region $\left(2250-2100 \mathrm{~cm}^{-1}\right.$, Figure 5 and Figures S12 and S13 in the Supporting Information) reveals that the zeolites possess some moderate and strong Lewis acidity (2200 and $2230 \mathrm{~cm}^{-1}$, respectively). ${ }^{51,52}$ These sites have been shown to induce cyclohexanone formation in the Beckmann rearrangement. ${ }^{53}$ Quantifying the values from the CO stretching peaks (Table S4 in the Supporting Information) and from the area values in the $\mathrm{NH}_{3}$-TPD (Table $\mathrm{S} 3$ in the Supporting Information) shows that the total quantity of acid sites follows the trend SAPO-37 > ZSM-5 > Zeolite-Y, despite similar dopant levels.

The QENS study has already shown that cyclohexanone oxime is able to access the internal sites of SAPO-37 and Zeolite-Y, but not ZSM-5. Although CO-probed FT-IR is able to probe the total acidity, a bulkier probe such as collidine was subsequently employed to identify selectively the acid sites that are accessible to the oxime. The latter should correspond to the vast majority of acid sites in the SAPO-37 and Zeolite-Y catalysts, but interaction can also be envisaged with acid sites located at the pore mouth and extremities of ZSM-5 (Figures S14-S19 in the Supporting Information). Both hydroxyl peaks in SAPO-37 and Zeolite-Y decrease on collidine adsorption, confirming that both supercage and sodalite cage protons are accessible: proton migration occurs at elevated temperatures (Figures S14, S16, and S18), or as collidine interacts with protons in the windows of the sodalite cages (most likely a through-space interaction, since collidine is too large to occupy these cages). Integrating the area of the collidine doublet at 1637 and $1652 \mathrm{~cm}^{-1}$ quantifies the number of accessible sites (Figures S15, S17, and S19 and Table 1). ${ }^{52}$ Despite ZSM-5 possessing a higher quantity of acid sites than Zeolite-Y (TPD and FTIR), the framework topology impedes access of the collidine probe and, consequently, only surface silanol species and those in the vicinity of the pore mouths are available for catalysis in ZSM-5 (Figures S15, S17, and S19). SAPO-37 and Zeolite-Y possess more accessible sites, and it was observed that, although SAPO-37 has a greater overall acidity (Table 1 and Figure 6), Zeolite- $Y$ has distinctly stronger acid sites, in agreement with $\mathrm{NH}_{3}$-TPD and low-temperature, $\mathrm{CO}$-probed FT-IR findings. ${ }^{15}$ The collidine results were in relatively good agreement with those obtained by $\mathrm{NH}_{3}$-TPD and those expected from the dopant loading (Table S5 in the Supporting Information). The total acidities from collidine FTIR were generally lower than those found experimentally through $\mathrm{NH}_{3}$ TPD; however, this is likely due to the difference in size between the probes, with the bulkier collidine probe blocking neighboring acid sites from other collidine molecules. ${ }^{5,7,8}$ The $\mathrm{NH}_{3}$-TPD for SAPO-37 is in excellent agreement with that predicted from the Si dopant loading (Table S5). ${ }^{5,7,8}$ The ZSM5 and Zeolite-Y $\mathrm{NH}_{3}$-TPD values are significantly lower than those predicted from $\mathrm{Al}$ dopant loading. This is likely due to aluminum islanding and the formation of nonisolated sites.

The spectroscopic data reinforce the importance of combining the desired active site with the appropriate

Table 1. Integrated Peak Areas and Calculated Acidities for the Collidine-Probed FT-IR

\begin{tabular}{|c|c|c|c|c|c|c|c|c|}
\hline \multirow[b]{2}{*}{ system } & \multicolumn{4}{|c|}{ integrated peak areas $(\mathrm{au} / \mathrm{mg})$} & \multicolumn{4}{|c|}{ total acidity $(\mathrm{mmol} / \mathrm{g})$} \\
\hline & weak & medium & strong & total & weak & medium & strong & total \\
\hline SAPO-37 & 0.913 & 2.845 & 1.609 & 5.367 & 0.070 & 0.219 & 0.124 & 0.413 \\
\hline Zeolite-Y & 0.625 & 0.806 & 1.259 & 2.690 & 0.048 & 0.062 & 0.097 & 0.207 \\
\hline ZSM-5 & 0.100 & 0.083 & 0.039 & 0.222 & 0.008 & 0.006 & 0.003 & 0.017 \\
\hline
\end{tabular}




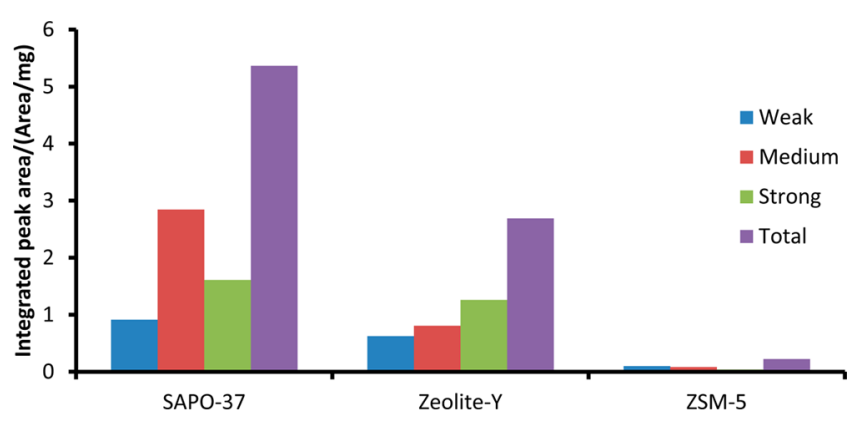

Figure 6. Summary of collidine FT-IR data for zeotype materials, highlighting the accessibility of the faujasite frameworks, with SAPO37 possessing a high fraction of medium-strength acid sites, whereas Zeolite-Y has a greater proportion of strong acid sites. The collidine probe is unable to access the ZSM-5 micropores, leading to significantly fewer acid sites.

framework topology, which is further exemplified in the observed catalytic trends for the liquid-phase Beckmann rearrangement. The catalytic data (Figure 7) show notable
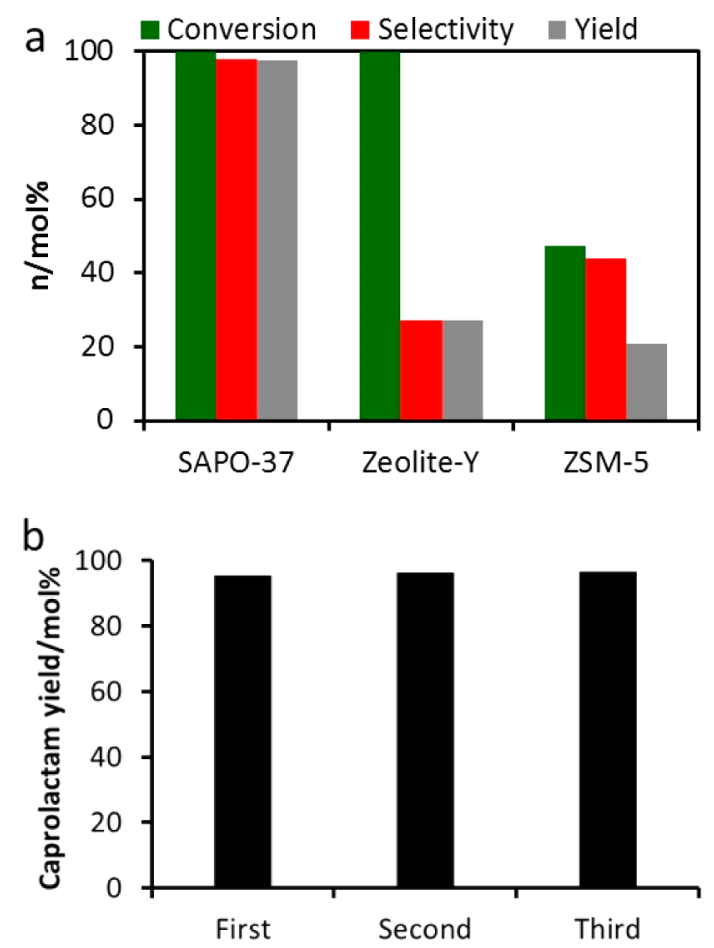

Figure 7. Liquid-phase Beckmann rearrangement data of cyclohexanone oxime: (a) highlighting the interplay among framework topology, active-site engineering, and molecular diffusion; (b) showing consistent caprolactam yields from SAPO-37 on multiple regenerations, vindicating the reusability of the catalyst. See the Supporting Information for experimental details.

trends that reflect the importance of the framework topology and acid-site strength, notwithstanding the critical role of diffusion and interaction of the substrate with the desired active site. The faujasitic frameworks (SAPO-37 and Zeolite-Y) show equivalent levels of conversion, which vastly exceed that of ZSM-5 (47 mol \%; Figure 7a). This trend is not commensurate with the total (measured) acidity (Figure S8 and Table S3 in the Supporting Information), and therefore other factors are responsible for this remarkable finding.
Despite having fewer acid sites than both ZSM-5 and SAPO37 , kinetic studies have shown that Zeolite-Y displays a superior rate of conversion of the cyclohexanone oxime substrate (Figure 8), although selectivity for caprolactam is low (Figure
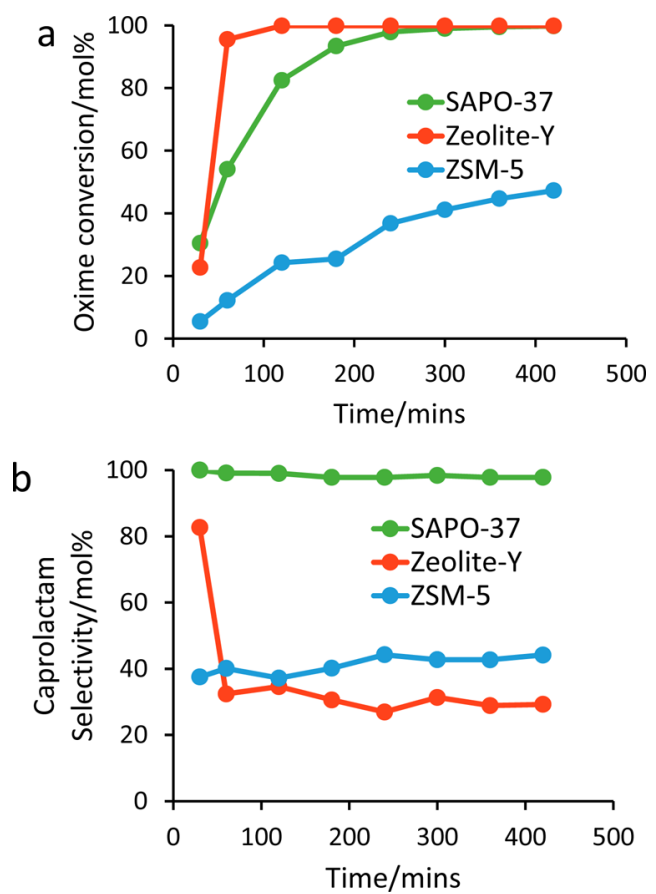

Figure 8. Liquid-phase Beckmann rearrangement data of cyclohexanone oxime showing the kinetic behavior of (a) conversion and (b) selectivity. See the Supporting Information for experimental details.

$8 b)$. These findings are noteworthy and justify our hypothesis that the "jump diffusion" observed in SAPO-37 originates from favorable catalyst-substrate interactions that are intrinsic to a specific acid site and independent of total acidity. The minimal Lewis acidity in the SAPO-37 species may also be a contributing factor; however, this does not contradict the QENS observations. ${ }^{51,52}$ The QENS results (Figure 2 and Figure S3 in the Supporting Information) unequivocally reveal that, unlike the faujasitic systems, cyclohexanone oxime cannot permeate into the micropores of ZSM-5 over the time scales investigated, further substantiating that catalytic selectivity to the desired product, caprolactam, relies primarily on accessible internal acid sites. ZSM-5 has accessible acid sites only on the surface and at the pore mouths, which impede both reactivity (because of hindered diffusion) and selectivity for $\varepsilon$ caprolactam (Scheme 1). As Zeolite-Y possesses a greater proportion of "stronger" acid sites (Table 1 and Figure 6) than SAPO-37, the formation of undesired side products is favored, ${ }^{53}$ in stark contrast to the (desired) weaker sites in SAPO-37. It is therefore remarkable that, while both Zeolite-Y and SAPO-37 display high conversions in the liquid-phase process (Figures $7 \mathrm{a}$ and $8 \mathrm{a}$ ), we did not observe any notable interactions with the oxime molecule in the QENS experiments for Zeolite-Y (Figure 2a and Figure S4 in the Supporting Information). These combined findings are therefore crucial in highlighting the importance of the favorable interactions of the internal acid sites in the SAPO-37 catalyst with the cyclohexanone oxime substrate, which lead to the highly selective formation of caprolactam in the liquid phase at low 
temperatures (Figures $7 \mathrm{a}$ and $8 \mathrm{~b}$ ). These internal acid sites are very stable and can be regenerated to deliver consistent caprolactam yields over several cycles, thereby confirming the recyclability and heterogeneity of the SAPO-37 catalyst (Figure $7 \mathrm{~b})$. The stability of the SAPO-37 catalyst was also evaluated postcatalysis. Powder XRD showed that the faujasitic crystalline structure was retained postcatalysis and on reactivation (Figure S20 in the Supporting Information). Similarly, $\mathrm{N}_{2}$ physisorption confirmed that the total pore volume of the material could be recovered on reactivation, with the micropore volume being completely retained (Table S6 in the Supporting Information). Finally, 1D ${ }^{27} \mathrm{Al}$ MAS NMR spectra (Figure S21 in the Supporting Information) showed no variation among fresh, postcatalysis, and reactivated SAPO-37 catalysts, either under nitrogen or on exposure to air for $12 \mathrm{~h}$. These findings, coupled with the catalytic data (multiple recycle tests, Figure $7 \mathrm{~b}$ ) demonstrate the stability of the SAPO-37 catalyst for this reaction.

These fundamental studies are of paramount importance in understanding the complex nature of the interplay among active site, framework topology, and reaction pathways. Understanding these subtle structure-activity relationships and associated diffusion pathways will pave the way for the industrial exploitation of the liquid-phase Beckmann rearrangement process, using heterogeneous solids. ${ }^{15}$

\section{CONCLUSIONS}

The ability to control precisely the nature and strength of solidacid sites, combined with significant insights into molecular diffusion behavior within nanoporous architectures, has led to important new insights into a novel catalytic system for the low-temperature, liquid-phase production of $\varepsilon$-caprolactam (precursor to Nylon-6). Neutron scattering (QENS and INS), in combination with aligned spectroscopic techniques, offers unique insights into the distinct role of surface and internal acid sites and their mechanistic influence in the liquidphase Beckmann rearrangement of cyclohexanone oxime with FAU and MFI catalysts. This study further elucidates the contrasting diffusion and catalytic pathways that are prevalent within these heterogeneous solids and highlights the interplay of framework topology and active site design in sustainable catalysis.

\section{ASSOCIATED CONTENT}

\section{S Supporting Information}

The Supporting Information is available free of charge on the ACS Publications website at DOI: 10.1021/acscatal.6b03641.

Full synthesis details, characterization data, and catalytic methodologies, basic QENS background theory, and further textural, neutron scattering, NMR, TPD, and FTIR data (PDF)

\section{AUTHOR INFORMATION}

\section{Corresponding Author}

*E-mail for R.R.: R.Raja@soton.ac.uk.

\section{ORCID $\odot$}

Stewart F. Parker: 0000-0002-3228-2570

Robert Raja: 0000-0002-4161-7053

Notes

The authors declare no competing financial interest.

\section{ACKNOWLEDGMENTS}

The UK Catalysis Hub is funded by the EPSRC (Engineering and Physical Sciences Research Council) via grants EP/ K014706/1, EP/K014668/1, EP/K014854/1, EP/K014714/1, and EP/M013219/1. The EPSRC also funded the Centre for Doctoral Training scheme with grant no. EP/G036675/1. The project was also funded by the Science and Technologies Facilities Council and ISIS Pulsed Neutron Muon Source. M.E.P., S.H.N., and S.C. also acknowledge Honeywell LLC for studentships. The UK Catalysis Hub is kindly thanked for resources and support provided via our membership in the UK Catalysis Hub Consortium. We thank the STFC Rutherford Appleton Laboratory and ISIS Pulsed Neutron and Muon Source for access to neutron beam facilities. Mr. Ryan Mead is also acknowledged for his assistance with catalyst synthesis.

\section{REFERENCES}

(1) Liebgott, P. P.; Leroux, F.; Burlat, B.; Dementin, S.; Baffert, C.; Lautier, T.; Fourmond, V.; Ceccaldi, P.; Cavazza, C.; Meynial-Salles, I.; Soucaille, P.; Fontecilla-Camps, J. C.; Guigliarelli, B.; Bertrand, P.; Rousset, M.; Léger, C. Nat. Chem. Biol. 2010, 6, 63-70.

(2) Forneris, F.; Mattevi, A. Science 2008, 321, 213-216.

(3) Jiang, J.; Jorda, J. L.; Yu, J.; Baumes, L. A.; Mugnaioli, E.; DiazCabanas, M. J.; Kolb, U.; Corma, A. Science 2011, 333, 1131-1134.

(4) Choi, M.; Na, K.; Kim, J.; Sakamoto, Y.; Terasaki, O.; Ryoo, R. Nature 2009, 461, 246-249.

(5) Raja, R.; Potter, M. E.; Newland, S. H. Chem. Commun. 2014, 50, 5940-5957.

(6) Choi, M.; Cho, H. S.; Srivastava, R.; Venkatesan, C.; Choi, D. H.; Ryoo, R. Nat. Mater. 2006, 5, 718-723.

(7) Potter, M. E.; Cholerton, M. E.; Kezina, J.; Bounds, R.; Carravetta, M.; Manzoli, M.; Gianotti, E.; Lefenfeld, M.; Raja, R. ACS Catal. 2014, 4, 4161-4169.

(8) Gianotti, E.; Manzoli, M.; Potter, M. E.; Shetti, V. N.; Sun, D.; Paterson, J.; Mezza, T. M.; Levy, A.; Raja, R. Chem. Sci. 2014, 5, 18101819.

(9) Dusselier, M.; Van Wouwe, P.; Dewaela, A.; Jacobs, P. A.; Sels, B. F. Science 2015, 349, 78-80.

(10) Holm, M.; Saravanamurugan, S.; Taarning, E. Science 2010, 328, 602-605.

(11) Leithall, R. M.; Shetti, V. N.; Maurelli, S.; Chiesa, M.; Gianotti, E.; Raja, R. J. Am. Chem. Soc. 2013, 135, 2915-2918.

(12) Paterson, J.; Potter, M. E.; Gianotti, E.; Raja, R. Chem. Commun. 2011, 47, 517-519.

(13) Buurmans, I. L. C.; Ruiz-Martínez, J.; Knowles, W. V.; van der Beek, D.; Bergwerff, J. A.; Vogt, E.T. C.; Weckhuysen, B. M. Nat. Chem. 2011, 3, 862-867.

(14) Lefenfeld, M.; Raja, R.; Paterson, A. J.; Potter, M. E. U.S. Patent US 9,012,709, 2015.

(15) Levy, A. B.; Raja, R.; Potter, M. E. U.S. Patent US 9,221,762, 2015.

(16) Maurelli, S.; Vishnuvarthan, M.; Chiesa, M.; Berlier, G.; Van Doorslaer, S. J. Am. Chem. Soc. 2011, 133, 7340-7343.

(17) Han, O. H.; Kim, C. S.; Hong, S. B. Angew. Chem., Int. Ed. 2002, 41, 469-472.

(18) Nylon-A global strategic business report; Global Industry Analysts Inc.: San Jose, CA, 2010.

(19) Chmelik, C.; Karger, J. Chem. Soc. Rev. 2010, 39, 4864-4884.

(20) Forni, L.; Fornasari, G.; Giordano, G.; Lucarelli, C.; Katovic, A.; Trifiro, F.; Perri, C.; Nagy, J. B. Phys. Chem. Chem. Phys. 2004, 6, $1842-1847$.

(21) Flego, C.; Dalloro, L. Microporous Mesoporous Mater. 2003, 60, 263-271.

(22) Izumi, Y.; Ichihashi, H.; Shimazu, Y.; Kitamura, M.; Sato, H. Bull. Chem. Soc. Jpn. 2007, 80, 1280-1287.

(23) Marie, O.; Thibault-Starzyk, F.; Massiani, P. J. Catal. 2005, 230, $28-37$. 
(24) Camblor, M. A.; Corma, A.; Garcia, H.; Semmer-Herldedan, V.; Valencia, S. J. Catal. 1998, 177, 267-272.

(25) Fernandez, A. B.; Lezcano-Gonzalez, I.; Boronat, M.; Blasco, T.; Corma, A. J. Catal. 2007, 249, 116-119.

(26) Heitmann, G. P.; Dahlhoff, G.; Hoelderich, W. F. J. Catal. 1999, 186, 12-19.

(27) Jobic, H.; Theodorou, D. N. Microporous Mesoporous Mater. 2007, 102, 21-50.

(28) O'Malley, A. J.; Parker, S. F.; Chutia, A.; Farrow, M. R.; Silverwood, I. P.; Garcia-Sakai, V.; Catlow, C. R. A. Chem. Commun. 2016, 52, 2897-2900.

(29) Schuster, C.; Hoelderich, W. F. Catal. Today 2000, 60, 193207.

(30) Treacey, M. M. J.; Higgins, J. B. Collection of simulated XRD powder patterns for Zeolites, 5th ed.; Elsevier Science and Technology: Amsterdam, 2007.

(31) Fick, A. Ann. Phys. 1855, 170, 59-86.

(32) van Hove, L. Phys. Rev. 1954, 95, 249-262.

(33) Jobic, H.; Fitch, A. N.; Combet, J. J. Phys. Chem. B 2000, 104, 8491-8497.

(34) Sahasrabudhe, A.; Mitra, S.; Tripathi, A. K.; Mukhopadhay, R.; Gupta, N. M. Phys. Chem. Chem. Phys. 2003, 5, 3066-3075.

(35) http://dx.doi.org/10.5286/SOFTWARE/MANTID.

(36) Mukhopadhyay, S. RAL Technical Report, RAL-TR-2014-005, 2014.

(37) Jobic, H.; Bee, M.; Pouget, S. J. Phys. Chem. B 2000, 104, 71307133.

(38) Auerbach, S. M.; Henson, N. J.; Cheetham, A. K.; Metiu, H. I. J. Phys. Chem. 1995, 99, 10600-10608.

(39) Segall, M. D.; Lindan, P. J. D.; Probert, M. J.; Pickard, C. J.; Hasnip, P. J.; Clark, S. J.; Payne, M. C. J. Phys.: Condens. Matter 2002, 14, 2717-2744.

(40) Clark, S. J.; Segall, M. D.; Pickard, C. J.; Hasnip, P. J.; Probert, M. J.; Refson, K.; Payne, M. C. Z. Kristallogr. - Cryst. Mater. 2005, 220, 567-570.

(41) Sastre, G.; Lewis, D. W.; Catlow, C. R. A. J. Phys. Chem. B 1997, 101, 5249-5262.

(42) Kobera, L.; Brus, J.; Klein, P.; Dedecek, J.; Urbanova, M. Solid State Nucl. Magn. Reson. 2014, 57-58, 29-38.

(43) Chen, T. H.; Wouters, B. H.; Grobet, P. J. J. Phys. Chem. B 1999, 103, 6179-6184.

(44) Fyfe, C. A.; Feng, Y.; Grondey, H.; Kokotailo, G. T.; Gies, H. Chem. Rev. 1991, 91, 1525-1543.

(45) Zhang, W.; Bao, X.; Guo, X.; Wang, X. Catal. Lett. 1999, 60, $89-94$.

(46) Jiao, J.; Kanellopoulos, J.; Wang, W.; Ray, S. S.; Foerster, H.; Freude, D.; Hunger, M. Phys. Chem. Chem. Phys. 2005, 7, 3221-3226.

(47) Rocha, J.; Carr, S. W.; Klinowski, J. Chem. Phys. Lett. 1991, 187, 401-408.

(48) Gianotti, E.; Bisio, C.; Marchese, L.; Guidotti, M.; Ravasio, N.; Psaro, P.; Coluccia, S. J. Phys. Chem. C 2007, 111, 5083-5089.

(49) Makarova, M. A.; Ojo, A. F.; Karim, K.; Hunger, M.; Dwyer, J. J. Phys. Chem. 1994, 98, 3619-3623.

(50) Bordiga, S.; Lamberti, C.; Geobaldo, F.; Zecchina, A.; Turnes Palomino, G.; Otero Arean, C. Langmuir 1995, 11, 527-533.

(51) Chakarova, K.; Hadjiivanov, K. J. Phys. Chem. C 2011, 115, $4806-4817$.

(52) Holm, M.; Svelle, S.; Joensen, F.; Beato, P.; Christensen, C. H.; Bordiga, S.; Bjorgen, M. Appl. Catal., A 2009, 356, 23-30.

(53) Ngamcharussrivichai, C.; Wu, P.; Tatsumi, T. J. Catal. 2005, $235,139-149$. 